\title{
Midiendo el desarrollo de República Dominicana desde el espacio
}

\author{
Liliana Cruz y EnriQue Penson*
}

Cómo citar: Cruz, L., \& Penson, E. (2018). Midiendo el desarrollo de República Dominicana desde el espacio. Ciencia, Economía y Negocios, 2(1), 63-91.

doi: http://dx.doi.org/10.22206/ceyn.2018.v2i1.pp63-91

\section{Resumen}

En este artículo se estima la relación entre la presencia de luces nocturnas en los municipios de República Dominicana y una serie de variables demográficas obtenidas de las bases de datos de los censos de 2002 y 2010. La información sobre la presencia de luces nocturnas se construyó a partir de las imágenes satelitales nocturnas anuales publicadas por la Administración Nacional Oceánica y Atmosférica (NOAA, por sus siglas en inglés) de Estados Unidos. Se encontró que existe una relación positiva y significativa entre la presencia de luces nocturnas y la población, la densidad poblacional, el acceso al tendido eléctrico público, el nivel de saneamiento y el índice de calidad de vida. De manera paralela, esta presencia de luces mantiene una fuerte correlación negativa con diferentes mediciones de la pobreza en cada municipio. Estos hallazgos son robustos a diferentes especificaciones econométricas y son congruentes con la literatura internacional. Se abre espacio en la República Dominicana para estudios posteriores que sirvan de complemento a la producción de estadísticas demográficas actualizadas, el ajuste de los marcos muestrales de los censos (para parametrizar mejor las encuestas oficiales en años donde no ocurren censos) y la identificación de cambios en las tendencias de indicadores de interés como la pobreza en tiempo real.

Palabras clave: luces nocturnas; desarrollo; República Dominicana.

JEL: E01, O11, O54, R23.

* Cruz, Liliana: Analytica. Dirección: c/ Boy Scout \#16, Ensanche Naco, Santo Domingo, República Dominicana. T: +18096835988 (email: lilianacruz703@gmail.com). Penson, Enrique: Analytica. Dirección: c/ Boy Scout \#16, Ensanche Naco, Santo Domingo, República Dominicana. T: +18096835988 (email: e.penson.brisindi@gmail.com) 


\title{
Measuring the Development of the Dominican Republic from Space
}

\author{
Liliana CRuz y EnRiQue Penson*
}

\begin{abstract}
This article estimates the correlation between the presence of night lights in the provinces of the Dominican Republic and demographic variables, obtained from the censuses realized in 1993, 2002 y 2010. The information relative to the presence of night lights was obtained from the data base of satellite nocturnal imagery published by the National Oceanic and Atmospheric Administration (NOAA). The results suggest a positive relation between nocturnal lights and the population, population density and the presence of a mature population (population in working ages). Moreover, these lights keep a strong negative correlation between the level of poverty of each province. The findings in this article are consistent with international literature and represent a pioneer study in Dominican Republic that precede a field of study that could be implemented to complement national demographic statistics, adjust the sample used for the census (in order to improve the polls in years when no censuses are lifted), and to identify changes in the trend of specials indicators as the poverty in real time.
\end{abstract}

JEL: E01, O11, O54, R23

Keywords: Nighttime lights, development, Dominican Republic

* Cruz, Liliana: Analytica. Address: c/ Boy Scout \#16, Ensanche Naco, Santo Domingo, Dominican Republic. F: +18096835988 (email: lilianacruz703@gmail.com). Penson, Enrique: Analytica. Address: c/ Boy Scout \#16, Ensanche Naco, Santo Domingo, Dominican Republic. F: +18096835988 (email: e.penson.brisindi@gmail.com) 


\section{Introducción}

Este trabajo de investigación propone que la utilización de data proveída libremente por el gobierno estadounidense sobre luces nocturnas puede servir para hacer una propuesta de un complemento a las estadísticas nacionales sobre indicadores demográficos, sociales y económicos en República Dominicana.

La producción de estadísticas demográficas, sociales y económicas es costosa y requiere de un largo período que ocupa recursos del Estado. A pesar de esto, los resultados de estos procesos de recopilación, estructuración, estimación y producción de datos tienen importantes externalidades positivas sobre las economías. En la medida en la que se cuenta con mejor información, los hogares, las empresas y el sector público pueden tomar decisiones informadas que maximicen el uso de los recursos limitados que poseen.

La información demográfica, particularmente, es un gran reto para cualquier Estado. La recolección de información sobre hogares y sus miembros implica la organización de grandes equipos de encuestadores y técnicos. La mayor expresión de esto es el censo poblacional, que en la República Dominicana se ha acordado hacer cada diez años; el último fue realizado en el año 2010. En el espacio temporal comprendido entre los censos se han hecho y se harán múltiples encuestas -ENFT, ENHOGAR, entre otras- que utilizan los marcos establecidos en los censos para derivar muestras y estimar indicadores sobre la población de República Dominicana; la posibilidad de incorporar el análisis de luces nocturnas permitiría hacer una propuesta, posterior a diversos estudios que trabajen el tema, que facilitaría la actualización de estos marcos muestrales.

El estudio utiliza las informaciones obtenidas desde los censos de 2010 y 2002 para estimar modelos indicativos de la relación entre la luminosidad en los diferentes municipios del país y los indicadores sociodemográficos como la población, la densidad poblacional, las distintas mediciones de pobreza y el acceso al tendido eléctrico. Se encuentran relaciones significativas en la mayoría de los casos, apoyando la tesis de que existe información valiosa dentro de las 
imágenes nocturnas de la República Dominicana que puede servir como insumo para el análisis de los cambios de los indicadores socioeconómicos de los municipios del país. Estos resultados deben considerarse dentro del contexto de un país en que el acceso a un servicio eléctrico de calidad y regular, disponible al grueso de la población, aún no es una meta totalmente cumplida, aunque durante las últimas dos décadas se ha logrado bastante.

El resto de este documento se ordena de la siguiente forma: en la sección 2 se describe la literatura relevante al tema en cuestión; en la sección 3 se discute sobre las fuentes de datos utilizadas, exponiendo sus fortalezas y debilidades; luego, en la sección 4, se trata la metodología de trabajo utilizada en el estudio; posteriormente se presentan los resultados econométricos en la sección 5; finalmente, en la sección 6 se concluye con un resumen de los hallazgos y se contempla la posibilidad de futuras investigaciones que tomen este documento como punto de partida.

\section{Revisión de la literatura}

Durante las últimas dos décadas ha surgido una serie de estudios que aprovechan la información libremente distribuida sobre luces nocturnas a través del mundo para complementar (o a veces incluso servir como fuente primaria) las estadísticas nacionales de diversas regiones del globo.

Elvidge, Baugh, Kihn, Kroehl, Davis \& Davis (1997) realizaron una de las primeras investigaciones en las que se relacionó la emisión de radiación observable, efectivamente luces nocturnas, con variables como la población, la actividad económica y el consumo de energía eléctrica, utilizando un corte transversal de 21 países. En el mismo año, Sutton, Roberts, Elvidge \& Meij (1997) demostraron, a nivel estatal y de condado, que las luces nocturnas pueden explicar el 63\% de la varianza de la densidad poblacional de Estados Unidos al año 1992.

Luego, Doll, Muller \& Elvidge (2000) probaron, ahora para una muestra de 46 países, la alta correlación y, por tanto, el valor predictivo de las luces nocturnas con el producto interno bruto (PIB) y 
la emisión de dióxido de carbono. Posteriormente, Elvidge, Imhoff, Baugh, Hobson, Nelson, Safran, Dietz y Tuttle (2001) comprobaron, ahora para una muestra de 200 naciones, la relación entre luminosidad y población, actividad económica, consumo de energía eléctrica y emisiones de carbono.

Por su parte, Amaral, Camara, Monteiro, Quintanilha y Elvidge (2005) mostraron la eficacia del uso de luces nocturnas para identificar correctamente la presencia de centros urbanos en Amazonia, junto con un poder predictivo de $79 \%$ sobre la población urbana del Estado de Pará. De modo similar, comprobaron la alta correlación entre la presencia de luces nocturnas y el consumo de energía eléctrica en la zona.

El trabajo de Ghosh, Anderson, Powell, Sutton y Elvidge (2009) va un paso más adelante que las investigaciones anteriores, estimando el volumen de la economía informal y las entradas de remesas internacionales en México. Encuentra que las estadísticas oficiales, a la vez, subestiman el tamaño de la economía informal en el país y que los ingresos por remesas podrían ser hasta un $50 \%$ más grandes de lo que indican las estadísticas oficiales.

Más adelante, Roychowdhury, Jones, Arrowsmith, Reinke y Bedford (2010) utilizaron la información del censo de 2001 de India, junto con data obtenida sobre las luces nocturnas observadas desde el espacio, para actualizar los indicadores del censo e incluso calcularlos para zonas geográficas más pequeñas que las que el censo permite identificar. Utilizan regresiones simples que logran explicar hasta el $90 \%$ de la varianza de diversos indicadores, tales como densidad poblacional, porcentaje de viviendas con vehículo, densidad poblacional urbana, entre otros.

Algunos trabajos como el de Henderson \& Storeygard (2012) incluso han desarrollado metodologías que permiten estimar elasticidades de largo plazo entre el nivel de luminosidad y el crecimiento económico medido a través del Producto Interno Bruto (PIB). Esto sentó las bases para la estimación de tasas de crecimiento en zonas geográficas no delimitadas por fronteras políticas, sino por accidentes geográficos, y en regiones que no cuentan con agencias estadísticas 
desarrolladas. Mostraron, por ejemplo, que las zonas costeras de África Sub-Sahariana están creciendo a un ritmo menor que las no costeras.

Para los interesados en una discusión mucho más amplia sobre el tratamiento de la información obtenida desde satélites para aplicaciones en el área de la economía, en el trabajo de Donaldson \& Storeygard (2016) se hace un recuento histórico de la evolución de estas técnicas, no solo enfocada en luces nocturnas, sino en la totalidad de informaciones disponibles desde satélites que toman mediciones del planeta.

Es importante notar que en algunos estudios, como en el de Addison \& Stewart (2015), se han notado dificultades en el uso de las luces nocturnas en modelos que relacionen estas con variables económicas como el Producto Interno Bruto (PIB), la producción manufacturera, el acervo de capital, el consumo de electricidad, la población total y la población urbana. La dificultad en la estimación responde a que los datos de luminosidad muestran cierto nivel de ruido y este hace más difícil determinar relaciones estadísticamente significativas entre la luminosidad y las variables económicas.

Los hallazgos mencionados anteriormente y la relativa novedad de este tipo de investigaciones sugieren que existe espacio para explorar esta línea de investigación en República Dominicana y complementar así la diversidad de estadísticas económicas, sociales y demográficas del país.

\section{Descripción de los datos}

La fuente primaria de información en este trabajo es la suministrada de forma abierta por la Administración Nacional Oceánica y Atmosférica (NOAA, por sus siglas en inglés) sobre la presencia e intensidad de las luces nocturnas observadas por satélites a través del año. Esta data es luego filtrada para eliminar el ruido causado por los fuegos forestales, el ciclo lunar, las estaciones, entre otros factores, y se guarda en un archivo digital desde 1992 que mantiene recurrencia hasta el presente -incluso con regularidad mensual posterior al 
2012-, con rezagos de actualización de no más de dos meses. Los archivos correspondientes al período pre-2012 se agrupan bajo las siglas DMSP y los posteriores (que tienen regularidad mensual) se concentran bajo las siglas VIIRS. La diferencia entre ambos es significativa, ya que el segundo tiene mayor precisión; los satélites que captaron los datos DMSP solo podrían discriminar cuadrículas de 5 $\mathrm{km}$ por $5 \mathrm{~km}\left(25 \mathrm{~km}^{2}\right)$, mientras que los VIIRS pueden discriminar cuadrículas de $742 \mathrm{~m}$ por $742 \mathrm{~m}\left(0.55 \mathrm{~km}^{2}\right)$, lo que significa que este segundo es 45 veces más preciso que el primero. Los archivos de la NOAA contienen, además de la información visual de luminosidad, el valor de medido por los satélites, ajustado estadísticamente para ubicarse entre 0 y $63^{1}$.

Este acercamiento inicial al análisis de las luces nocturnas en República Dominicana solo utilizará los archivos anuales desde 1993 hasta 2012, debido, principalmente, a que la información posterior a 2012 (mensual) se encuentra en un formato diferente producto de cambios metodológicos y en los satélites de recolección de datos ${ }^{2}$. También, este primer estudio trabaja con datos de los censos de población de República Dominicana de 2002 y 2012; esto pretende intentar que el primer acercamiento a esta línea de investigación en el país tome como referencia los datos más abarcadores de la población, que son los de los censos, antes de embarcarse en el tratamiento de datos obtenidos a partir de encuestas, que son recogidos desde muestras.

Las figuras que aparecen debajo muestran las luces nocturnas obtenidas desde los archivos de 1993 y 2012, respectivamente, en República Dominicana, con las delimitaciones de las diferentes provincias marcadas. Se nota que estas luces revelan claramente la posición de los principales centros urbanos del país, como es el caso de la capital, en el sur de la isla, y Santiago, en el norte. También se advierte un crecimiento en la mayoría de las zonas, con atención especial a la

\footnotetext{
${ }^{1}$ Ver Elvidge, C., Baugh, K., Zhizhin, M. \& Chi, F. (2013), para una mayor discusión sobre las propiedades técnicas.

${ }^{2}$ Se abre el espacio a otros trabajos de consolidación estadística de las series 1993-2012 y de 2012 en adelante. Sin embargo, debe notarse que este esfuerzo no ha sido logrado aún, ni siquiera por parte de los técnicos de la NOAA.
} 
aparición (desde prácticamente nada) de una presencia importante de luces en el polo este extremo de la isla, probablemente relacionado con el acelerado desarrollo turístico de la zona.

De los archivos obtenidos a partir de la NOAA es posible calcular una luminosidad promedio por provincia (mostrada en la tabla debajo) $\mathrm{y}$, de esta forma, verificar que el centro urbano del país coincide con altos valores de luminosidad promedio históricamente, como se nota en el caso de la capital (Distrito Nacional) y la provincia que la rodea (Santo Domingo). Las provincias con mayor crecimiento en el período 19932010 han sido La Altagracia, Santiago Rodríguez, Samaná y San Juan, como se puede observar en la tabla debajo. Se nota también que estas provincias con mayor crecimiento iniciaron con una baja "dotación" de luminosidad, por lo que se hace necesario comprobar si existe una relación significativa entre la dotación inicial y el crecimiento, lo que apoyaría la hipótesis de convergencia entre la luminosidad de las provincias; la siguiente figura muestra que efectivamente existe una relación significativa y negativa entre la dotación inicial de las provincias en el año 1993 y su eventual crecimiento durante el período 1993-2012. 
Figura 1. LuCes nocturnas en República Dominicana (1993 y 2012)

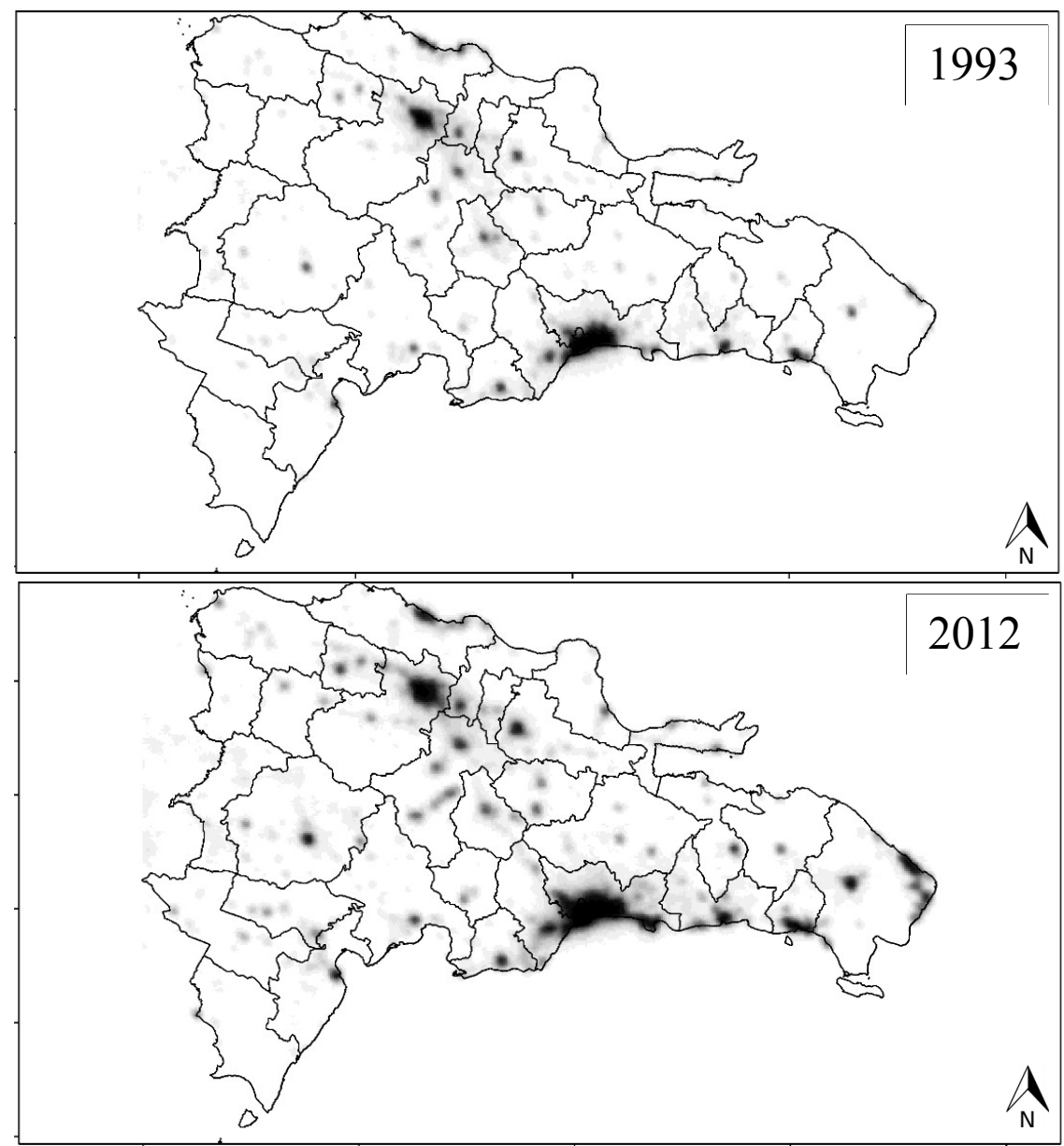

Nota: esta figura muestra imágenes de las luces nocturnas observadas desde el espacio en 1993 (arriba) y 2012 (debajo), construidas con datos obtenidos a partir de la Administración Nacional Oceánica y Atmosférica (NOAA, por sus siglas en inglés). Las delimitaciones de las diferentes provincias del país están marcadas en los gráficos. 
Tabla 1. Luminosidad PROMEdio en las PROVINCIAS de República Dominicana 1993-2012

\begin{tabular}{|c|c|c|c|c|c|c|}
\hline Provincia & 1993 & 2002 & 2010 & $\begin{array}{c}\text { Var. } \\
\text { 1993-2002 }\end{array}$ & $\begin{array}{c}\text { Var. } \\
\text { 2002-2010 }\end{array}$ & $\begin{array}{c}\text { Var. } \\
1993-2010\end{array}$ \\
\hline Azua & 0.83 & 1.38 & 1.40 & $67 \%$ & $1 \%$ & $69 \%$ \\
\hline Bahoruco & 0.71 & 2.05 & 2.07 & $186 \%$ & $1 \%$ & $190 \%$ \\
\hline Barahona & 1.05 & 1.94 & 2.28 & $85 \%$ & $18 \%$ & $118 \%$ \\
\hline Dajabón & 0.31 & 0.82 & 0.71 & $167 \%$ & $-14 \%$ & $130 \%$ \\
\hline Distrito Nacional & 58.19 & 61.24 & 62.32 & $5 \%$ & $2 \%$ & $7 \%$ \\
\hline Duarte & 1.61 & 4.34 & 3.58 & $170 \%$ & $-18 \%$ & $122 \%$ \\
\hline El Seybo & 0.34 & 1.06 & 0.95 & $209 \%$ & $-10 \%$ & $178 \%$ \\
\hline Espaillat & 3.42 & 6.81 & 4.80 & $99 \%$ & $-29 \%$ & $40 \%$ \\
\hline Hato Mayor & 0.60 & 1.95 & 1.88 & $223 \%$ & $-3 \%$ & $212 \%$ \\
\hline Independencia & 0.38 & 0.88 & 0.94 & $133 \%$ & $6 \%$ & $148 \%$ \\
\hline La Altagracia & 0.80 & 3.07 & 4.78 & $284 \%$ & $56 \%$ & $498 \%$ \\
\hline La Estrelleta & 0.55 & 0.58 & 1.19 & $5 \%$ & $105 \%$ & $116 \%$ \\
\hline La Romana & 4.89 & 9.64 & 10.33 & $97 \%$ & $7 \%$ & $112 \%$ \\
\hline La Vega & 2.54 & 5.98 & 5.06 & $136 \%$ & $-15 \%$ & $99 \%$ \\
\hline María Trinidad Sánchez & 0.69 & 2.26 & 1.47 & $227 \%$ & $-35 \%$ & $113 \%$ \\
\hline Monseñor Nouel & 3.30 & 5.64 & 3.71 & $71 \%$ & $-34 \%$ & $12 \%$ \\
\hline Monte Cristi & 0.43 & 1.04 & 0.97 & $140 \%$ & $-6 \%$ & $126 \%$ \\
\hline Monte Plata & 0.58 & 1.52 & 1.55 & $160 \%$ & $2 \%$ & $165 \%$ \\
\hline Pedernales & 0.19 & 0. & & $24 \%$ & $37 \%$ & $70 \%$ \\
\hline Peravia & 3.49 & 5.56 & 5.76 & $59 \%$ & $4 \%$ & $65 \%$ \\
\hline Puerto Plata & 2.23 & 4.47 & 3.21 & $101 \%$ & $-28 \%$ & $44 \%$ \\
\hline Sánchez Ramírez & 1.37 & 2.51 & 2.70 & $83 \%$ & $8 \%$ & $96 \%$ \\
\hline Salcedo & 2.48 & 5.30 & 3.81 & $114 \%$ & $-28 \%$ & $54 \%$ \\
\hline Samaná & 0.53 & 2.10 & 2.50 & $294 \%$ & $19 \%$ & $369 \%$ \\
\hline San Cristóbal & 5.22 & 8.91 & 10.39 & $71 \%$ & $17 \%$ & $99 \%$ \\
\hline San José de Ocoa & 0.67 & 1.28 & 1.42 & $90 \%$ & $11 \%$ & $111 \%$ \\
\hline San Juan & 0.58 & 1.07 & 2.10 & $85 \%$ & $97 \%$ & $264 \%$ \\
\hline San Pedro de Macorís & 3.61 & 7.26 & 6.93 & $101 \%$ & $-5 \%$ & $92 \%$ \\
\hline Santiago Rodríguez & 0.25 & 0.91 & 1.25 & $262 \%$ & $37 \%$ & $397 \%$ \\
\hline Santiago & 4.52 & 7.65 & 7.10 & $69 \%$ & $-7 \%$ & $57 \%$ \\
\hline Santo Domingo & 13.07 & 22.14 & 23.83 & $69 \%$ & $8 \%$ & $82 \%$ \\
\hline Valverde & 1.77 & 4.12 & 4.85 & $133 \%$ & $18 \%$ & $174 \%$ \\
\hline País Completo & 1.89 & 3.68 & 3.75 & $95 \%$ & $2 \%$ & $98 \%$ \\
\hline
\end{tabular}

Nota: esta figura muestra la luminosidad promedio por provincia en el período 1993-2010 y el crecimiento de varios períodos, construido a partir de datos obtenidos desde la NOAA. 
La siguiente figura muestra la relación entre la variación de la luminosidad en todas las provincias del país en el período 1993-2010 (eje vertical) y el nivel de luminosidad en cada provincia en el año 1993.

Figura 2. Relación entre la VARIACión de la Luminosidad EN 1993-2010 Y SU VALOR EN 1993

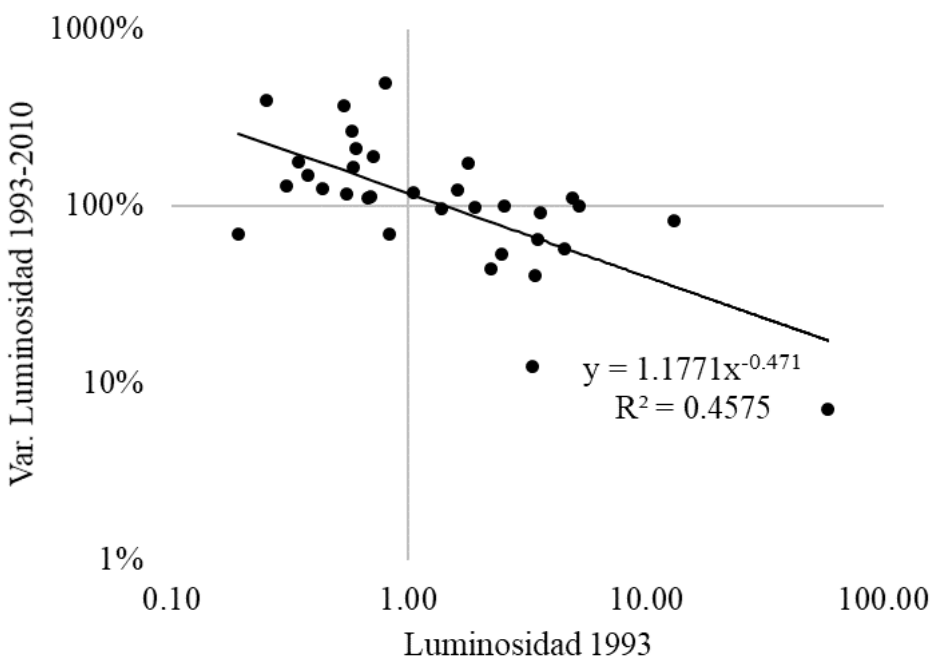

Fuente: La figura fue construida con datos obtenidos a partir de la Administración Nacional Oceánica y Atmosférica (NOAA, por sus siglas en inglés). Se muestra la ecuación estimada de la relación entre las variables con el indicador de bondad de ajuste ( $\mathrm{r}$ cuadrado) debajo.

Las fuentes secundarias de información en este trabajo son las informaciones sobre los censos de 2002 y 2010, publicadas de forma abierta por parte de la Oficina Nacional de Estadística (ONE) de la República Dominicana y por el Ministerio de Economía, Planificación y Desarrollo (MEPyD). De aquí se extrajeron, a nivel municipal, los indicadores como población total, población por km2, desocupación, acceso al tendido eléctrico público, porcentaje de personas en el grupo socioeconómico muy bajo, bajo y muy bajo más bajo, porcentaje de hogares en condiciones de hacinamiento, índice de calidad de vida y porcentaje de hogares con alto grado de saneamiento.

Debajo se encuentra una tabla que denota el nombre abreviado que se utilizará para referirse a las variables de ahora en adelante durante 
las secciones de descripción de datos y resultados de los ejercicios de inferencia estadística.

TABla 2. NOMENClatura DE VARIABLES UTILIZADAS

\begin{tabular}{ll}
\hline Identificador & Significado \\
\hline Ln(Media) & Logaritmo Natural de la Luminosidad Promedio \\
Ln(Std. Dev.) & Logaritmo Natural de la Desviación Estándar de la Luminosidad \\
pob & Población \\
pob.km ${ }^{2}$ & Población por km² \\
desocup & Desocupación \\
tenido & Acceso al Tendido Eléctrico Público \\
gse_mb & \% de Personas en el Grupo Socioeconómico Muy Bajo \\
gse_b & \% de Personas en el Grupo Socioeconómico Bajo \\
gse_mbb & \% de Personas en el Grupo Socioeconómico Muy Bajo y Bajo \\
hacinamiento & Porcentaje de Personas viviendo en Hacinamiento \\
ICV & índice de Calidad de Vida \\
saneamiento & Porcentaje de Personas viviendo con Alto Saneamiento \\
\hline
\end{tabular}

Nota: esta tabla muestra el identificador de cada variable a utilizar, con su descripción.

La tabla debajo muestra las estadísticas descriptivas de las variables calculadas u obtenidas a partir de los datos de la ONE y el MEPyD, por municipio. Se nota que el municipio promedio tiene 57,000 habitantes, con una densidad poblacional de 361 personas por $\mathrm{km}^{2}$; sin embargo, la desviación estándar de ambos indicadores es significativa, siendo más de dos veces la media, en el caso de la población, y más de tres veces la media, en el caso de la densidad poblacional. La desocupación promedio se ubica en un $12 \%$, pero también con gran desviación; el municipio con menor desocupación en la muestra tiene un valor de $2.7 \%$, mientras que el de mayor desocupación tiene un valor de $32 \%$. El acceso al tendido eléctrico es bastante disperso también, oscilando entre $7.4 \%$ y $99.8 \%$, con una media de $87 \%$. Más de la mitad de las personas en cada municipio (52\%), en promedio, pertenecen a los grupos socioeconómicos muy bajo o bajo, siendo la mayoría de estos pertenecientes al bajo; la presencia de grupos socioeconómicos muy bajos y/o bajos muestra, al igual que las demás variables, gran dispersión. El porcentaje de personas que vive en condiciones de hacinamiento, en promedio, en los diferentes municipios se ubica en el $28 \%$, oscilando entre un mínimo de $11.3 \%$ y $59.3 \%$. El 
índice de calidad de vida promedio en la muestra tiene un valor promedio de $56.7 \%$, con una desviación estándar de $9.1 \%$, variando entre un mínimo de $31.7 \%$ y un máximo de $87.6 \%$. El porcentaje de personas viviendo en condiciones de alto saneamiento es relativamente bajo, en promedio, con un valor de $19.4 \%$, con una alta dispersión, oscilando entre un mínimo de $0.3 \%$ y un máximo de $76.8 \%$.

Tabla 3. RESUmen ESTAdístico de LaS VARIABLES DEMOGRÁFICAS POR MUNICIPIO

\begin{tabular}{lccccc}
\hline Statistic & N & Mean & St. Dev. & Min & Max \\
\hline pob & 310 & $56,653.7$ & $125,817.7$ & 2,470 & 965,040 \\
pob.km2 & 310 & 361.0 & $1,130.6$ & 6.4 & $10,535.4$ \\
desocup & 310 & 12.0 & 4.9 & 2.7 & 32.3 \\
tendido & 310 & 87.2 & 14.0 & 7.4 & 99.8 \\
gse.mb & 310 & 18.3 & 11.9 & 1.9 & 63.0 \\
gse.b & 310 & 33.7 & 8.0 & 10.4 & 54.6 \\
gse.mbb & 310 & 52.0 & 16.6 & 12.4 & 92.9 \\
hacinamiento & 310 & 28.0 & 9.4 & 11.3 & 59.3 \\
ICV & 310 & 56.7 & 9.1 & 31.7 & 87.6 \\
saneamiento & 310 & 19.3 & 14.2 & 0.3 & 76.8 \\
\hline
\end{tabular}

Nota: esta tabla muestra las estadísticas descriptivas de los datos utilizados en esta investigación, construidos a partir de informaciones de la NOAA y la ONE.

A continuación, se visualizan todas las variables (en nivel y logaritmos) extraídas de las bases de datos de los censos de 2002 y 2010 , graficadas contra el logaritmo natural de la media de luminosidad en cada provincia en el período en cuestión, extraídas de las informaciones suministradas por la NOAA. A simple vista, se observa una relación positiva entre la población y la población por $\mathrm{km}^{2}$ (en nivel y logaritmo) con la luminosidad promedio. También, se identifica una relación negativa entre la luminosidad y la tasa de pobreza general (en nivel y logaritmo). Para el resto de las variables, a nivel visual no se aprecia una relación clara, aunque la estimación estadística en secciones posteriores confirmará estos hechos. 
FigURA 3. VARIABLES EN NIVEL CONTRA LUMINOSIDAD PROMEDIO POR MUNICIPIO
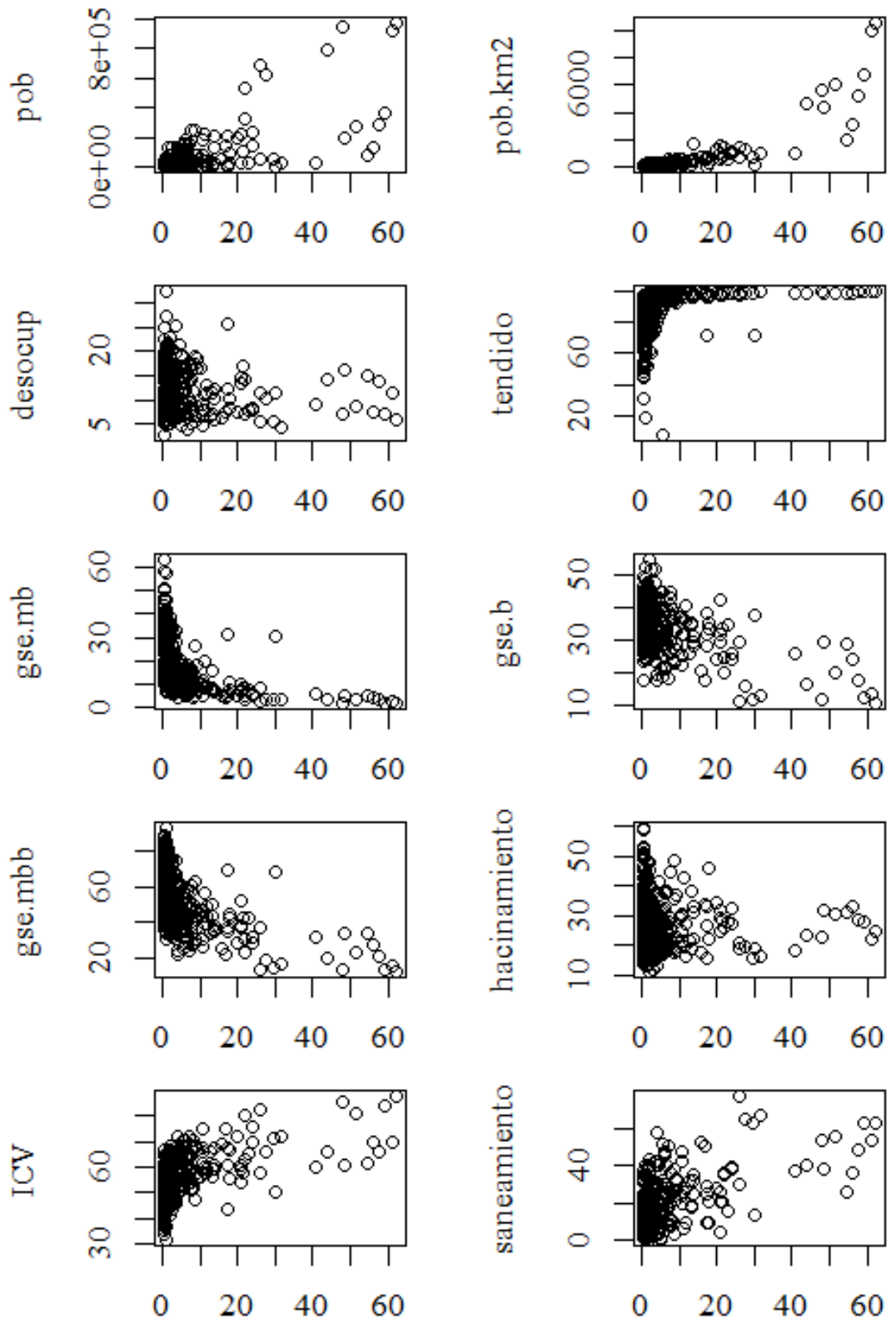

Nota: esta figura muestra todas las variables demográficas (en nivel) extraídas de las bases de datos del censo de 2002 y 2010, visualizadas contra el logaritmo natural de la luminosidad promedio de cada municipio. Fue construida a partir de las informaciones de la NOAA y la ONE. 
Figura 4. Logaritmo de Variables CONTRa logaritmo de LUMinOSIDAD PROMEDIO POR MUNICIPIO

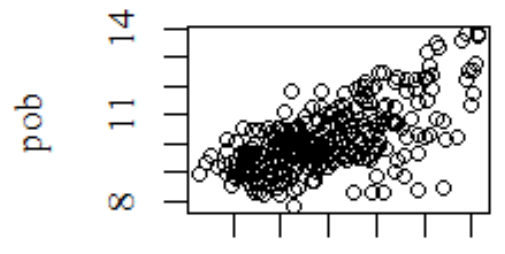

$\begin{array}{lll}-1 & 1 & 3\end{array}$

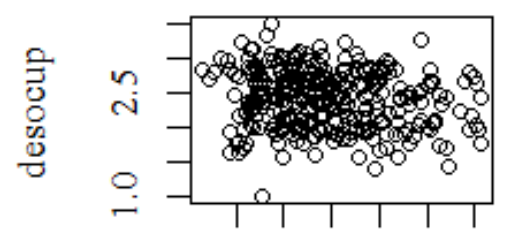

$\begin{array}{lll}-1 & 1 & 3\end{array}$

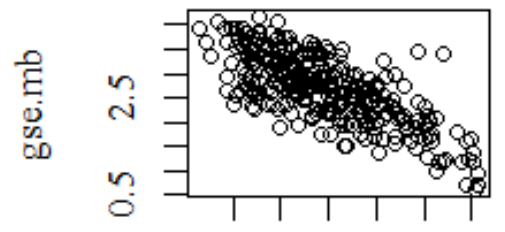

$\begin{array}{lll}-1 & 1 & 3\end{array}$

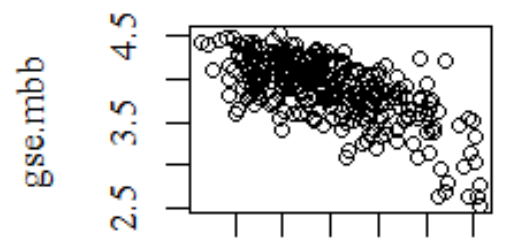

$\begin{array}{lll}-1 & 1 & 3\end{array}$

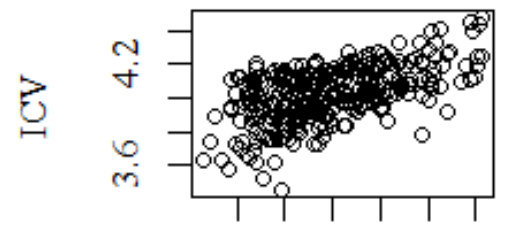

$\begin{array}{lll}-1 & 1 & 3\end{array}$

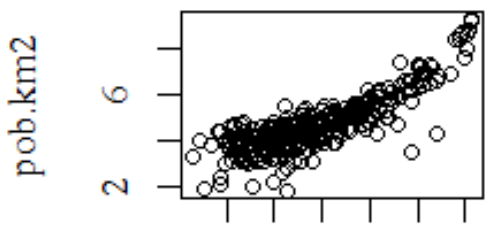

$\begin{array}{lll}-1 & 1 & 3\end{array}$

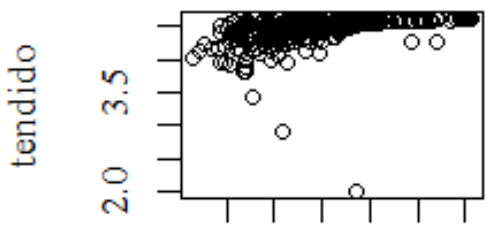

$\begin{array}{lll}-1 & 1 & 3\end{array}$

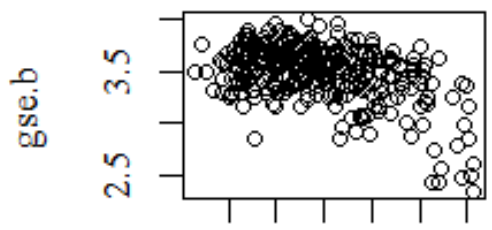

$\begin{array}{lll}-1 & 1 & 3\end{array}$

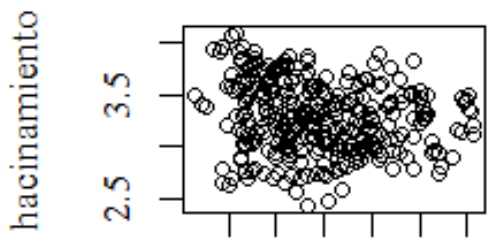

$\begin{array}{lll}-1 & 1 & 3\end{array}$

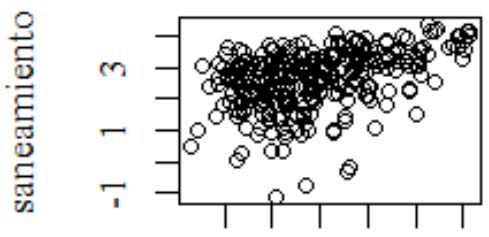

$\begin{array}{lll}-1 & 1 & 3\end{array}$

Esta figura muestra todas las variables demográficas (en logaritmo natural) extraídas de las bases de datos del censo de 2002 y 2010, visualizadas contra el logaritmo natural de la luminosidad promedio de cada municipio. Fue construida a partir de las informaciones de la NOAA y la ONE. 


\section{Metodología utilizada}

La metodología utilizada, en congruencia con la literatura revisada, implica realizar una serie de regresiones lineales utilizando como variables explicativas la media y la desviación estándar de la luminosidad dentro de cada demarcación geográfica -en este caso, los municipios-.

Todas las variables, explicadas y explicativas, se introducirán en el modelo luego de pasar por una transformación logarítmica. Esta decisión responde principalmente a que se teme la presencia de heterocedasticidad en los modelos, lo que invalidaría las pruebas estadísticas que verifican si los coeficientes estimados son estadísticamente diferentes de cero.

También, se agregará a las ecuaciones estimadas variables dicotómicas del año del censo, para así tratar de aislar la variación de los indicadores, que simplemente responde al avance del tiempo.

En el caso de que se detecte la presencia de heterocedasticidad en las ecuaciones estimadas, se reestimarían con errores estándares robustos. De esta forma, se verificaría si aun ampliando los intervalos de confianza asociados a los coeficientes estimados, estos permanecen estadísticamente significativos.

Finalmente, se estimarán ecuaciones separadas para cada año en la muestra para, primero, verificar la estabilidad de los coeficientes, y segundo, detectar si la especificación propuesta ha perdido o ha ganado poder explicativo a través del tiempo. También, se incluirá la presencia de una variable dicotómica, como forma de una prueba de robustez, que describe si el municipio en cuestión pertenece a las provincias Distrito Nacional, Santo Domingo o Santiago (grandes centros urbanos del país) y se interaccionará con las demás variables independientes para validar si esta parte de la muestra contiene valores tan extremos que sesgan la estimación de los coeficientes. 


\section{Resultados}

\section{Estimaciones simples}

Los resultados de la batería de ecuaciones estimadas fueron mixtos. En la tabla 4 se verifica que en los modelos de las variables población (1), población por $\mathrm{km}^{2}$ (2), tendido eléctrico (4), diferentes medidas de pobreza $(5,6,7)$, hacinamiento (8), ICV (9) y saneamiento (10), se encuentra una relación significativa con las luces nocturnas. El modelo que relaciona la desocupación con diferentes indicadores asociados a la luminosidad no muestra un buen ajuste, más allá del logrado por la inclusión de una variable dicotómica que identifica si el valor corresponde al año 2010, en vez de al 2002.

\section{Población}

Se encuentra que el aumento en el promedio de luminosidad está asociado, al menos inicialmente, a la disminución de la expectativa de población, pero eventualmente (debido al componente cuadrático) se relaciona con el aumento de la población; de manera análoga, la desviación estándar de la luminosidad dentro de la provincia está asociada al aumento de la población. La densidad poblacional, medida como población por $\mathrm{km}^{2}$, aumenta lineal y cuadráticamente con el promedio de luminosidad y disminuye con la desviación estándar.

\section{Acceso al tendido eléctrico público, saneamiento y hacina- miento}

Se encontró, como se esperaba, que el aumento de la luminosidad promedio está asociado al aumento del acceso al tendido eléctrico público. El aumento de la luminosidad promedio está asociado, inicialmente, a la disminución del hacinamiento y el saneamiento, pero eventualmente el término cuadrático lo afecta positivamente. La desviación estándar de la luminosidad está positivamente asociada al saneamiento.

\section{Pobreza e Índice de Calidad de Vida}

Se encontró que el aumento de la luminosidad promedio está asociado negativamente a las diferentes mediciones de la pobreza, tanto 
en forma lineal como cuadrática, mientras que la desviación estándar de la luminosidad está negativamente asociada a la pobreza. El ICV (Índice de Calidad de Vida) está positivamente asociado al cuadrado de la luminosidad promedio y a la desviación estándar de la luminosidad en el municipio.

TABla 4. Resultados de ESTIMACiONES Simples

\begin{tabular}{lcccccccccc}
\hline & $\begin{array}{c}\text { Pob. } \\
(1)\end{array}$ & $\begin{array}{c}\text { Pob.Km2 } \\
(2)\end{array}$ & $\begin{array}{c}\text { Desocup } \\
(3)\end{array}$ & $\begin{array}{c}\text { Tendido } \\
(4)\end{array}$ & $\begin{array}{c}\text { G.MB } \\
(5)\end{array}$ & $\begin{array}{c}\text { G.B } \\
(6)\end{array}$ & $\begin{array}{c}\text { G.MBB } \\
(7)\end{array}$ & $\begin{array}{c}\text { Hacinamiento } \\
(8)\end{array}$ & $\begin{array}{c}\text { ICV } \\
(9)\end{array}$ & $\begin{array}{c}\text { Saneamiento } \\
(10)\end{array}$ \\
\hline Ln(Media) & $-0.62^{* * *}$ & $0.62^{* * *}$ & -0.03 & $0.07^{* *}$ & $-0.17^{* * *}$ & $0.08^{* * *}$ & -0.01 & $-0.10^{* *}$ & 0.004 & $-0.30^{* * *}$ \\
& $(0.11)$ & $(0.09)$ & $(0.04)$ & $(0.03)$ & $(0.07)$ & $(0.03)$ & $(0.03)$ & $(0.05)$ & $(0.01)$ & $(0.11)$ \\
Ln(Media)'Sqr & $0.22^{* * *}$ & $0.13^{* * *}$ & -0.01 & -0.01 & $-0.05^{* * *}$ & $-0.06^{* * *}$ & $-0.05^{* * *}$ & $0.02^{* *}$ & $0.01^{* * *}$ & $0.12^{* * *}$ \\
& $(0.03)$ & $(0.02)$ & $(0.01)$ & $(0.01)$ & $(0.02)$ & $(0.01)$ & $(0.01)$ & $(0.01)$ & $(0.003)$ & $(0.03)$ \\
Ln(Std. Dev.) $)$ & $0.92^{* * *}$ & $-0.18^{* * *}$ & -0.01 & 0.03 & $-0.13^{* *}$ & $-0.06^{* *}$ & $-0.09^{* * *}$ & -0.002 & $0.06^{* * *}$ & $0.44^{* * *}$ \\
& $(0.08)$ & $(0.07)$ & $(0.03)$ & $(0.03)$ & $(0.05)$ & $(0.02)$ & $(0.03)$ & $(0.04)$ & $(0.01)$ & $(0.09)$ \\
Año 2010 & $0.27^{* * *}$ & $0.17^{* *}$ & $-0.63^{* * *}$ & $0.07^{* * *}$ & $-0.33^{* * *}$ & $-0.23^{* * *}$ & $-0.27^{* * *}$ & 0.05 & $0.21^{* * *}$ & $0.63^{* * *}$ \\
& $(0.08)$ & $(0.07)$ & $(0.03)$ & $(0.02)$ & $(0.05)$ & $(0.02)$ & $(0.03)$ & $(0.04)$ & $(0.01)$ & $(0.08)$ \\
Constante & $9.03^{* * *}$ & $3.95^{* * *}$ & $2.77^{* * *}$ & $4.34^{* * *}$ & $3.27^{* * *}$ & $3.73^{* * *}$ & $4.25^{* * *}$ & $3.28^{* * *}$ & $3.83^{* * *}$ & $1.85^{* * *}$ \\
& $(0.08)$ & $(0.06)$ & $(0.03)$ & $(0.02)$ & $(0.05)$ & $(0.02)$ & $(0.02)$ & $(0.03)$ & $(0.01)$ & $(0.08)$ \\
$N$ & 310 & 310 & 310 & 310 & 310 & 310 & 310 & 310 & 310 & 310 \\
$\mathrm{R}^{2}$ & 0.59 & 0.77 & 0.61 & 0.17 & 0.60 & 0.50 & 0.63 & 0.05 & 0.72 & 0.35 \\
Adjusted R $\mathrm{R}^{2}$ & 0.59 & 0.77 & 0.60 & 0.16 & 0.60 & 0.49 & 0.63 & 0.04 & 0.71 & 0.34 \\
F Statistic & $11.11^{* * *} 260.84^{* * *} 118.03^{* * *} 16.00^{* *} 114.87^{* * *}$ & $75.96^{* * *} 129.97^{* * *}$ & $4.32^{* * *}$ & $192.73^{* * *}$ & $41.55^{* * *}$ \\
\hline
\end{tabular}

Nota: esta tabla muestra los resultados de la estimación de la serie de ecuaciones planteada en la sección de metodología. Cada modelo cuenta con el coeficiente asociado a la variable, con asteriscos mostrando el nivel de significancia estadística asociado; "*” denota significancia al 10\%, “**” al 5\% y “***” al 1\%). Debajo de los coeficientes estimados se encuentra el error estándar asociado al coeficiente. Debajo se muestra el número de observaciones $(\mathrm{N})$, los coeficientes de ajuste estadístico ( $\mathrm{R}^{2}$ y $\mathrm{R}^{2}$ Ajustado) y el estadístico F de cada modelo.

\section{Pruebas de heterocedasticidad}

Se verificó la existencia potencial de heterocedasticidad en la mayoría de las ecuaciones estimadas, como se puede observar en la tabla debajo, que muestra el estadístico de Breusch-Pagan, los grados de libertad (DF, por sus siglas en inglés) y el valor probabilístico asociado a la prueba. En todas las ecuaciones, exceptuando las de población por $\mathrm{km}^{2}$, acceso al tendido eléctrico, porcentaje de hogares muy pobres y saneamiento, se rechaza la hipótesis de no heterocedasticidad, con un $99 \%$ de confianza. En el caso de la ecuación de saneamiento, se rechaza la hipótesis de no heterocedasticidad, con un $98 \%$ de confianza. 
Tabla 5. Pruebas de heterocedasticidad de las estimaciones simples

\begin{tabular}{lccc}
\hline \multicolumn{1}{c}{ Variable } & $\begin{array}{c}\text { Estadístico de } \\
\text { Breusch-Pagan }\end{array}$ & $\begin{array}{c}\text { Grados de Liber- } \\
\text { tad (DF) }\end{array}$ & $\begin{array}{c}\text { Valor Probabilísti- } \\
\text { co (p-value) }\end{array}$ \\
\hline Población & 49.11 & 4.00 & 0.00 \\
Población por Km ${ }^{2}$ & 6.29 & 4.00 & 0.18 \\
Desocupación & 22.26 & 4.00 & 0.00 \\
Tendido Eléctrico & 1.64 & 4.00 & 0.80 \\
GSE_MB & 5.55 & 4.00 & 0.24 \\
GSE_B & 38.69 & 4.00 & 0.00 \\
GSE_MBB & 49.67 & 4.00 & 0.00 \\
Hacinamiento & 23.05 & 4.00 & 0.00 \\
ICV & 25.16 & 4.00 & 0.00 \\
Saneamiento & 11.25 & 4.00 & 0.02 \\
\hline
\end{tabular}

Esta tabla muestra el valor probabilístico asociado a la hipótesis nula de la prueba de Breusch-Pagan para los modelos estimados (Tabla 2). La hipótesis nula es que los errores del modelo estimado evidencian la presencia de heterocedasticidad.

\section{Estimaciones con errores estándares robustos}

La presencia de heterocedasticidad en los modelos compromete la validez de las pruebas de hipótesis, permitiendo así la posibilidad de que algunos de los coeficientes que se juzgaron significativos no lo sean realmente. Sin embargo, aun utilizando errores estándares robustos, que aíslan el sesgo a sobre-rechazar la hipótesis nula de que los coeficientes sean estadísticamente iguales a cero, las variables que inicialmente resultaron significativas permanecen así, como se observa en la tabla debajo. 
TABLA 6. MOdElos ESTIMAdOS CON ERRORES ESTÁNDARES ROBUSTOS

\begin{tabular}{lccccccccccc}
\hline & $\begin{array}{c}\text { Pob. } \\
\end{array}$ & Pob.Km2 Desocup Tendido & G.MB & \multicolumn{2}{c}{ G.B } & \multicolumn{2}{c}{ G.MBB } \\
& $(1)$ & $(2)$ & $(3)$ & $(4)$ & $(5)$ & $(6)$ & $(7)$ & $(8)$ & $(9)$ & $(10)$ \\
\hline Ln(Media) & $-0.62^{* * *}$ & $0.62^{* * *}$ & -0.03 & $0.07^{* * *}$ & $-0.17^{* * *}$ & $0.08^{* * *}$ & -0.01 & $-0.10^{* *}$ & 0.004 & $-0.30^{* * *}$ \\
& $(0.11)$ & $(0.10)$ & $(0.04)$ & $(0.03)$ & $(0.07)$ & $(0.03)$ & $(0.03)$ & $(0.05)$ & $(0.01)$ & $(0.11)$ \\
Ln(Media)'Sqr & $0.22^{* * *}$ & $0.13^{* * *}$ & -0.01 & -0.01 & $-0.05^{* * *}$ & $-0.06^{* * *}$ & $-0.05^{* * *}$ & $0.02^{* *}$ & $0.01^{* * *}$ & $0.12^{* * *}$ \\
& $(0.03)$ & $(0.02)$ & $(0.01)$ & $(0.01)$ & $(0.01)$ & $(0.01)$ & $(0.01)$ & $(0.01)$ & $(0.003)$ & $(0.02)$ \\
Ln(Std. Dev.) $)$ & $0.92^{* * *}$ & $-0.18^{* *}$ & -0.01 & 0.03 & $-0.13^{* *}$ & $-0.06^{* *}$ & $-0.09^{* * *}$ & -0.002 & $0.06^{* * *}$ & $0.44^{* * *}$ \\
& $(0.12)$ & $(0.07)$ & $(0.03)$ & $(0.02)$ & $(0.05)$ & $(0.03)$ & $(0.03)$ & $(0.04)$ & $(0.01)$ & $(0.08)$ \\
Año 2010 & $0.27^{* * *}$ & $0.17^{* *}$ & $-0.63^{* * *}$ & $0.07^{* * *}$ & $-0.33^{* * *}$ & $-0.23^{* * *}$ & $-0.27^{* * *}$ & 0.05 & $0.21^{* * *}$ & $0.63^{* * *}$ \\
& $(0.08)$ & $(0.07)$ & $(0.03)$ & $(0.02)$ & $(0.05)$ & $(0.02)$ & $(0.03)$ & $(0.04)$ & $(0.01)$ & $(0.09)$ \\
Constante & $9.03^{* * *}$ & $3.95^{* * *}$ & $2.77^{* * *}$ & $4.34^{* * *}$ & $3.27^{* * *}$ & $3.73^{* * *}$ & $4.25^{* * *}$ & $3.28^{* * *}$ & $3.83^{* * *}$ & $1.85^{* * *}$ \\
& $(0.07)$ & $(0.06)$ & $(0.03)$ & $(0.02)$ & $(0.04)$ & $(0.02)$ & $(0.02)$ & $(0.04)$ & $(0.01)$ & $(0.09)$ \\
\hline
\end{tabular}

Esta tabla muestra los resultados de la estimación de las ecuaciones, con errores estándares robustos para aislar el efecto de la presencia de heterocedasticidad detectada. Cada modelo cuenta con el coeficiente asociado a la variable, con asteriscos mostrando el nivel de significancia estadística asociado; “*” denota significancia al 10\%, “**” al 5\% y “***” al 1\%).

\section{Pruebas de robustez}

Como forma de realizar pruebas de robustez sobre los modelos propuestos, se estimaron dos especificaciones adicionales. Primero, la inclusión de una variable dicotómica que indica si el municipio pertenece a las provincias Santo Domingo, Distrito Nacional o Santiago, probando si la presencia de estas grandes ciudades sesga los coeficientes estimados. Segundo, se estimaron las ecuaciones de manera separada para cada año en la muestra y se compararon con la estimación original.

La tabla debajo muestra el efecto de la inclusión de la variable dicotómica que se refiere a grandes ciudades. Se encontró que la variable dicotómica es significativa, tanto en nivel por sí sola como al hacer interacción con las otras variables del modelo (luminosidad promedio, luminosidad promedio al cuadrado y desviación estándar de la luminosidad. Esto significa que la presencia de grandes centros urbanos dentro de la muestra podría sesgar el valor de los coeficientes estimados. 
Tabla 7. Modelos estimados con InClusión de Variable dicotómica de GRANDES CIUDADES

\begin{tabular}{|c|c|c|c|c|c|c|c|c|c|c|}
\hline & $\begin{array}{l}\text { Pob. } \\
\text { (1) }\end{array}$ & $\begin{array}{c}\text { Pob.Km2 } \\
\text { (2) }\end{array}$ & $\begin{array}{c}\text { Desocup } \\
\text { (3) }\end{array}$ & $\begin{array}{c}\text { Tendido } \\
\text { (4) }\end{array}$ & $\begin{array}{l}\text { G.MB } \\
(5)\end{array}$ & $\begin{array}{l}\text { G.B } \\
(6)\end{array}$ & $\begin{array}{c}\text { G.MBB } \\
(7)\end{array}$ & $\begin{array}{l}\text { Hacinamiento } \\
\text { (8) }\end{array}$ & $\begin{array}{c}\text { ICV } \\
(9)\end{array}$ & $\begin{array}{c}\text { Saneamiento } \\
(10)\end{array}$ \\
\hline Ln(Media) & $\begin{array}{c}-0.60^{\text {***** }} \\
(0.10)\end{array}$ & $\begin{array}{c}0.70^{* * * * *} \\
(0.11)\end{array}$ & $\begin{array}{l}-0.03 \\
(0.05)\end{array}$ & $\begin{array}{l}0.07^{* * *} \\
(0.03)\end{array}$ & $\begin{array}{c}-0.20^{\text {***** }} \\
(0.07)\end{array}$ & $\begin{array}{l}0.08^{* * * *} \\
(0.02)\end{array}$ & $\begin{array}{l}-0.02 \\
(0.03)\end{array}$ & $\begin{array}{l}-0.10^{*} \\
(0.05)\end{array}$ & $\begin{array}{l}0.004 \\
(0.02)\end{array}$ & $\begin{array}{c}-0.35^{* * *} \\
(0.12)\end{array}$ \\
\hline$->\mathrm{GC}$ & $\begin{array}{c}-0.79^{* *} \\
(0.35)\end{array}$ & $\begin{array}{l}-0.43 \\
(0.32)\end{array}$ & $\begin{array}{l}-0.01 \\
(0.11)\end{array}$ & $\begin{array}{l}0.005 \\
(0.04)\end{array}$ & $\begin{array}{l}0.48^{* *} \\
(0.20)\end{array}$ & $\begin{array}{c}0.05 \\
(0.11)\end{array}$ & $\begin{array}{c}0.18 \\
(0.12)\end{array}$ & $\begin{array}{l}0.20^{* * *} \\
(0.09)\end{array}$ & $\begin{array}{l}-0.05^{*} \\
(0.03)\end{array}$ & $\begin{array}{l}-0.03 \\
(0.23)\end{array}$ \\
\hline Ln(Media)'Sqr & $\begin{array}{c}0.16^{* * *} \\
(0.03)\end{array}$ & $\begin{array}{c}0.09^{* * *} \\
(0.03)\end{array}$ & $\begin{array}{l}-0.004 \\
(0.01)\end{array}$ & $\begin{array}{l}-0.01 \\
(0.01)\end{array}$ & $\begin{array}{l}-0.03 \\
(0.02)\end{array}$ & $\begin{array}{c}-0.03^{* * *} \\
(0.01)\end{array}$ & $\begin{array}{c}-0.02^{* * * *} \\
(0.01)\end{array}$ & $\begin{array}{c}0.04^{* * *} \\
(0.01)\end{array}$ & $\begin{array}{c}0.01 \\
(0.004)\end{array}$ & $\begin{array}{c}0.11^{* * * *} \\
(0.03)\end{array}$ \\
\hline$->\mathrm{GC}$ & $\begin{array}{c}0.29^{* * * *} \\
(0.06)\end{array}$ & $\begin{array}{l}0.14^{* *} \\
(0.06)\end{array}$ & $\begin{array}{l}0.003 \\
(0.02)\end{array}$ & $\begin{array}{l}-0.002 \\
(0.01)\end{array}$ & $\begin{array}{c}-0.12^{* * * *} \\
(0.04)\end{array}$ & $\begin{array}{l}-0.04^{*} \\
(0.02)\end{array}$ & $\begin{array}{c}-0.07^{* * *} \\
(0.03)\end{array}$ & $\begin{array}{l}-0.04^{*} \\
(0.02)\end{array}$ & $\begin{array}{c}0.02^{* * *} \\
(0.01)\end{array}$ & $\begin{array}{c}0.03 \\
(0.05)\end{array}$ \\
\hline Ln(Std. Dev.) & $\begin{array}{c}0.99^{* * *} \\
(0.08)\end{array}$ & $\begin{array}{c}-0.22^{* * *} \\
(0.08)\end{array}$ & $\begin{array}{l}-0.02 \\
(0.04)\end{array}$ & $\begin{array}{c}0.03 \\
(0.03)\end{array}$ & $\begin{array}{c}-0.13^{* *} \\
(0.06)\end{array}$ & $\begin{array}{c}-0.08^{* * * *} \\
(0.02)\end{array}$ & $\begin{array}{c}-0.12^{* * * *} \\
(0.03)\end{array}$ & $\begin{array}{l}-0.01 \\
(0.04)\end{array}$ & $\begin{array}{c}0.07^{* * *} \\
(0.01)\end{array}$ & $\begin{array}{c}0.51^{* * *} \\
(0.10)\end{array}$ \\
\hline$->\mathrm{GC}$ & $\begin{array}{l}-0.27 \\
(0.35)\end{array}$ & $\begin{array}{c}0.29 \\
(0.21)\end{array}$ & $\begin{array}{c}0.06 \\
(0.05)\end{array}$ & $\begin{array}{l}-0.01 \\
(0.03)\end{array}$ & $\begin{array}{c}0.01 \\
(0.14)\end{array}$ & $\begin{array}{l}0.12^{*} \\
(0.07)\end{array}$ & $\begin{array}{c}0.12 \\
(0.09)\end{array}$ & $\begin{array}{c}0.07 \\
(0.06)\end{array}$ & $\begin{array}{l}-0.04 \\
(0.02)\end{array}$ & $\begin{array}{c}-0.41^{* * *} \\
(0.15)\end{array}$ \\
\hline Año 2010 & $\begin{array}{c}0.28^{* * * *} \\
(0.08)\end{array}$ & $\begin{array}{c}0.18^{* * *} \\
(0.07)\end{array}$ & $\begin{array}{c}-0.63^{* * *} \\
(0.03)\end{array}$ & $\begin{array}{c}0.07^{* * * *} \\
(0.02)\end{array}$ & $\begin{array}{c}-0.34^{* * * *} \\
(0.05)\end{array}$ & $\begin{array}{c}-0.23^{* * * *} \\
(0.02)\end{array}$ & $\begin{array}{c}-0.27^{* * * *} \\
(0.02)\end{array}$ & $\begin{array}{c}0.05 \\
(0.04)\end{array}$ & $\begin{array}{c}0.21^{* * *} \\
(0.01)\end{array}$ & $\begin{array}{c}0.64^{* * *} \\
(0.08)\end{array}$ \\
\hline $\mathrm{GC}$ & $\begin{array}{l}0.50^{* *} \\
(0.23)\end{array}$ & $\begin{array}{c}-0.66^{* * * *} \\
(0.16)\end{array}$ & $\begin{array}{c}-0.23^{* * *} \\
(0.10)\end{array}$ & $\begin{array}{c}0.04 \\
(0.03)\end{array}$ & $\begin{array}{c}-0.46^{* * * *} \\
(0.10)\end{array}$ & $\begin{array}{c}-0.23^{* * * *} \\
(0.08)\end{array}$ & $\begin{array}{c}-0.35^{* * * *} \\
(0.07)\end{array}$ & $\begin{array}{c}-0.61^{* * * *} \\
(0.06)\end{array}$ & $\begin{array}{c}0.07^{* * * *} \\
(0.02)\end{array}$ & $\begin{array}{l}0.95^{* * * *} \\
(0.17)\end{array}$ \\
\hline Constante & $\begin{array}{c}9.04^{* * *} \\
(0.07)\end{array}$ & $\begin{array}{c}3.99^{* * *} \\
(0.06)\end{array}$ & $\begin{array}{c}2.77^{* * *} \\
(0.03)\end{array}$ & $\begin{array}{l}4.34^{* * *} \\
(0.02)\end{array}$ & $\begin{array}{c}3.26^{* * *} \\
(0.05)\end{array}$ & $\begin{array}{c}3.72^{* * *} \\
(0.02)\end{array}$ & $\begin{array}{l}4.25^{* * *} \\
(0.02)\end{array}$ & $\begin{array}{c}3.29^{* * * *} \\
(0.04)\end{array}$ & $\begin{array}{c}3.83^{* * *} \\
(0.01)\end{array}$ & $\begin{array}{l}1.83^{* * *} \\
(0.09)\end{array}$ \\
\hline
\end{tabular}

Nota: esta tabla muestra los resultados de la estimación de las ecuaciones, con errores estándares robustos para aislar el efecto de la presencia de heterocedasticidad detectada. Cada modelo cuenta con el coeficiente asociado a la variable, con asteriscos mostrando el nivel de significancia estadística asociado; “*” denota significancia al 10\%, “**” al 5\% y “***" al $1 \%$. Debajo de las variables asociadas a la luminosidad se encuentre el término "->GC", que indica la interacción de la variable superior con la variable dicotómica que toma valor 1 cuando el municipio pertenece a Santo Domingo, Santiago o el Distrito Nacional.

Las sub-secciones debajo muestran la estimación de las diferentes ecuaciones propuestas, incluyendo la presencia de la variable dicotómica indicativa de grandes ciudades, para la muestra completa y luego separando por años, como parte de las pruebas de robustez de las estimaciones.

\section{Población}

Como se puede observar debajo, la estimación de los modelos de población y densidad poblacional en las sub-muestras de 2002 y 2010 obtuvo resultados mixtos. Una serie de coeficientes se hicieron más pequeños en términos absolutos, como es el caso del promedio de luminosidad en el modelo de población $(-0.63$ a -0.56) y densidad 
poblacional (0.73 a 0.65$)$, al igual que el término cuadrático en la ecuación de población $(0.18$ a 0.15$)$ y el coeficiente asociado a la desviación estándar en esta misma última ecuación (1.02 a 0.96). Es posible que esto refleje un deterioro en la sensibilidad de estas variables en la medida que avanza el tiempo.

Tabla 8. Prueba de robustez por años de modelos de población

\begin{tabular}{|c|c|c|c|c|c|c|}
\hline & \multicolumn{3}{|c|}{ Población } & \multicolumn{3}{|c|}{ Población por Km2 } \\
\hline & $\begin{array}{l}\text { Total } \\
\text { (1) }\end{array}$ & $\begin{array}{c}2002 \\
(2) \\
\end{array}$ & $\begin{array}{c}2010 \\
(3) \\
\end{array}$ & $\begin{array}{c}\text { Total } \\
(4)\end{array}$ & $\begin{array}{c}2002 \\
(5) \\
\end{array}$ & $\begin{array}{c}2010 \\
(6) \\
\end{array}$ \\
\hline Ln(Media) & $\begin{array}{r}-0.60^{* * *} \\
(0.10)\end{array}$ & $\begin{array}{r}-0.63^{* * *} \\
(0.14)\end{array}$ & $\begin{array}{c}-0.56^{* * *} \\
(0.14)\end{array}$ & $\begin{array}{c}0.70^{* * *} \\
(0.11)\end{array}$ & $\begin{array}{c}0.73^{* * *} \\
(0.16)\end{array}$ & $\begin{array}{r}0.65^{* * *} \\
(0.14)\end{array}$ \\
\hline$->\mathrm{GC}$ & $\begin{array}{r}-0.79^{* *} \\
(0.35)\end{array}$ & $\begin{array}{l}-0.94 \\
(0.60)\end{array}$ & $\begin{array}{r}-0.78^{* *} \\
(0.34)\end{array}$ & $\begin{array}{c}-0.43 \\
(0.32)\end{array}$ & $\begin{array}{l}-0.73 \\
(0.60)\end{array}$ & $\begin{array}{l}-0.09 \\
(0.33)\end{array}$ \\
\hline Ln(Media)'Sqr & $\begin{array}{r}0.16^{* * *} \\
(0.03)\end{array}$ & $\begin{array}{r}0.18^{* * *} \\
(0.05)\end{array}$ & $\begin{array}{c}0.15^{* * *} \\
(0.04)\end{array}$ & $\begin{array}{c}0.09^{* * *} \\
(0.03)\end{array}$ & $\begin{array}{c}0.07 \\
(0.05)\end{array}$ & $\begin{array}{r}0.11^{* * *} \\
(0.04)\end{array}$ \\
\hline$->\mathrm{GC}$ & $\begin{array}{c}0.29^{* * *} \\
(0.06)\end{array}$ & $\begin{array}{l}0.28^{* *} \\
(0.11)\end{array}$ & $\begin{array}{c}0.31^{* * *} \\
(0.07)\end{array}$ & $\begin{array}{l}0.14^{* *} \\
(0.06)\end{array}$ & $\begin{array}{l}0.21^{*} \\
(0.11)\end{array}$ & $\begin{array}{l}0.07 \\
(0.07)\end{array}$ \\
\hline Ln(Std. Dev.) & $\begin{array}{l}0.99^{* * *} \\
(0.08)\end{array}$ & $\begin{array}{r}1.02^{* * *} \\
(0.12)\end{array}$ & $\begin{array}{c}0.96^{* * *} \\
(0.11)\end{array}$ & $\begin{array}{r}-0.22^{* * *} \\
(0.08)\end{array}$ & $\begin{array}{l}-0.2 \\
(0.12)\end{array}$ & $\begin{array}{r}-0.24^{* *} \\
(0.10)\end{array}$ \\
\hline 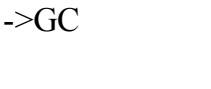 & $\begin{array}{l}-0.27 \\
(0.35)\end{array}$ & $\begin{array}{l}-0.07 \\
(0.62)\end{array}$ & $\begin{array}{l}-0.37 \\
(0.37)\end{array}$ & $\begin{array}{l}0.29 \\
(0.21)\end{array}$ & $\begin{array}{c}0.42 \\
(0.46)\end{array}$ & $\begin{array}{l}0.21 \\
(0.16)\end{array}$ \\
\hline 2010 & $\begin{array}{r}0.28^{* * *} \\
(0.08)\end{array}$ & & & $\begin{array}{r}0.18^{* * *} \\
(0.07)\end{array}$ & & \\
\hline $\mathrm{GC}$ & $\begin{array}{l}0.50^{* *} \\
(0.23)\end{array}$ & $\begin{array}{l}0.31 \\
(0.41)\end{array}$ & $\begin{array}{l}0.66^{* *} \\
(0.27)\end{array}$ & $\begin{array}{c}-0.66^{* * *} \\
(0.16)\end{array}$ & $\begin{array}{c}-0.73^{* *} \\
(0.36)\end{array}$ & $\begin{array}{r}-0.74^{* * *} \\
(0.14)\end{array}$ \\
\hline Constante & $\begin{array}{l}9.04^{* * *} \\
(\cap \cap 7)\end{array}$ & $\begin{array}{c}9.02^{* * *} \\
(\cap \cap 8)\end{array}$ & $\begin{array}{l}9.33^{* * *} \\
(\cap \cap 7)\end{array}$ & $\begin{array}{l}3.99^{* * *} \\
(\cap \cap 6)\end{array}$ & $\begin{array}{l}4.00^{* * *} \\
(\cap \text { no) }\end{array}$ & $\begin{array}{l}4.16^{* * *} \\
(\cap \cap 7)\end{array}$ \\
\hline
\end{tabular}

Nota: esta tabla muestra los resultados de la estimación de las ecuaciones, con errores estándares robustos para aislar el efecto de la presencia de heterocedasticidad detectada. Cada modelo cuenta con el coeficiente asociado a la variable, con asteriscos mostrando el nivel de significancia estadística asociado; "** denota significancia al 10\%, “**” al 5\% y “***" al 1\%. Debajo de las variables asociadas a la luminosidad se encuentra el término "->GC", que indica la interacción de la variable superior con la variable dicotómica que toma valor 1 cuando el municipio pertenece a Santo Domingo, Santiago o el Distrito Nacional. 


\section{Acceso al tendido eléctrico público, saneamiento y hacina- miento}

La tabla que veremos a continuación muestra que la separación de las muestras por años (2002) revela que algunos modelos, como el de acceso al tendido eléctrico público, ya no son significativos en el 2010; el coeficiente atado al promedio de la luminosidad en cada municipio pasa de un valor de 0.10 , pudiendo afirmar que es diferente de cero con un $99 \%$ de confianza, a un valor de 0.02 , que es estadísticamente igual a cero (no se rechaza la hipótesis nula). Otros modelos, como el de saneamiento, muestran que el valor de todos los coeficientes disminuye, evidenciando que el nivel de saneamiento esperado es menos sensible a la luminosidad en 2010 que en 2002. El efecto de la separación de la muestra en el modelo de hacinamiento es similar a los dos anteriores; el coeficiente atado al promedio de luminosidad pierde totalmente su significancia y el coeficiente atado al cuadrado del promedio de luminosidad pasa de ser significativo con un $99 \%$ de confianza a serlo apenas con un $90 \%$. 
Tabla 9. Prueba de robustez por años de modelos de tendido, hacinamiento y SANEAMIENTO

\begin{tabular}{|c|c|c|c|c|c|c|c|c|c|}
\hline & \multicolumn{3}{|c|}{ Tendido } & \multicolumn{3}{|c|}{ Hacinamiento } & \multicolumn{3}{|c|}{ Saneamiento } \\
\hline & $\begin{array}{c}\text { Total } \\
(1) \\
\end{array}$ & $\begin{array}{c}2002 \\
(2) \\
\end{array}$ & $\begin{array}{c}2010 \\
(3) \\
\end{array}$ & $\begin{array}{c}\text { Total } \\
(4) \\
\end{array}$ & $\begin{array}{c}2002 \\
(5) \\
\end{array}$ & $\begin{array}{c}2010 \\
(6) \\
\end{array}$ & $\begin{array}{c}\text { Total } \\
(7) \\
\end{array}$ & $\begin{array}{c}2002 \\
(8) \\
\end{array}$ & $\begin{array}{c}2010 \\
(9) \\
\end{array}$ \\
\hline Ln(Media) & $\begin{array}{r}0.07^{* *} \\
(0.03)\end{array}$ & $\begin{array}{r}0.10 * * * \\
(0.03)\end{array}$ & $\begin{array}{l}0.02 \\
(0.06)\end{array}$ & $\begin{array}{r}-0.10^{*} \\
(0.05)\end{array}$ & $\begin{array}{l}-0.08 \\
(0.07)\end{array}$ & $\begin{array}{c}-0.11 \\
(0.09)\end{array}$ & $\begin{array}{r}-0.35^{* * *} \\
(0.12)\end{array}$ & $\begin{array}{r}-0.46^{* *} \\
(0.20)\end{array}$ & $\begin{array}{r}-0.26^{* *} \\
(0.13)\end{array}$ \\
\hline$->\mathrm{GC}$ & $\begin{array}{l}0.005 \\
(0.04)\end{array}$ & $\begin{array}{l}-0.03 \\
(0.07)\end{array}$ & $\begin{array}{l}0.03 \\
(0.06)\end{array}$ & $\begin{array}{r}0.20^{* *} \\
(0.09)\end{array}$ & $\begin{array}{l}0.16 \\
(0.11)\end{array}$ & $\begin{array}{l}0.25^{*} \\
(0.14)\end{array}$ & $\begin{array}{c}-0.03 \\
(0.23)\end{array}$ & $\begin{array}{l}-0.15 \\
(0.44)\end{array}$ & $\begin{array}{l}0.16 \\
(0.25)\end{array}$ \\
\hline Ln(Media)'Sqr & $\begin{array}{l}-0.01 \\
(0.01)\end{array}$ & $\begin{array}{r}-0.01 * \\
(0.01)\end{array}$ & $\begin{array}{c}0.001 \\
(0.01)\end{array}$ & $\begin{array}{r}0.04 * * * \\
(0.01)\end{array}$ & $\begin{array}{l}0.04 * \\
(0.02)\end{array}$ & $\begin{array}{l}0.04^{*} \\
(0.02)\end{array}$ & $\begin{array}{r}0.11 * * * \\
(0.03)\end{array}$ & $\begin{array}{r}0.13^{* *} \\
(0.05)\end{array}$ & $\begin{array}{r}0.09 * * * \\
(0.03)\end{array}$ \\
\hline$->\mathrm{GC}$ & $\begin{array}{r}-0.002 \\
(0.01)\end{array}$ & $\begin{array}{l}0.004 \\
(0.01)\end{array}$ & $\begin{array}{l}-0.01 \\
(0.01)\end{array}$ & $\begin{array}{r}-0.04 * \\
(0.02)\end{array}$ & $\begin{array}{c}-0.04 \\
(0.03)\end{array}$ & $\begin{array}{c}-0.04 \\
(0.03)\end{array}$ & $\begin{array}{l}0.03 \\
(0.05)\end{array}$ & $\begin{array}{l}0.08 \\
(0.10)\end{array}$ & $\begin{array}{l}-0.03 \\
(0.05)\end{array}$ \\
\hline Ln(Std. Dev.) & $\begin{array}{l}0.03 \\
(0.03)\end{array}$ & $\begin{array}{l}0.04 \\
(0.03)\end{array}$ & $\begin{array}{l}0.03 \\
(0.04)\end{array}$ & $\begin{array}{l}-0.01 \\
(0.04)\end{array}$ & $\begin{array}{l}-0.07 \\
(0.05)\end{array}$ & $\begin{array}{l}0.04 \\
(0.06)\end{array}$ & $\begin{array}{r}0.51 * * * \\
(0.10)\end{array}$ & $\begin{array}{r}0.68 * * * \\
(0.16)\end{array}$ & $\begin{array}{r}0.36 * * * \\
(0.11)\end{array}$ \\
\hline$->\mathrm{GC}$ & $\begin{array}{l}-0.01 \\
(0.03)\end{array}$ & $\begin{array}{l}0.002 \\
(0.05)\end{array}$ & $\begin{array}{l}-0.03 \\
(0.04)\end{array}$ & $\begin{array}{l}0.07 \\
(0.06)\end{array}$ & $\begin{array}{l}0.14 * \\
(0.08)\end{array}$ & $\begin{array}{l}0.01 \\
(0.08)\end{array}$ & $\begin{array}{r}-0.41 * * * \\
(0.15)\end{array}$ & $\begin{array}{c}-0.50^{*} \\
(0.30)\end{array}$ & $\begin{array}{r}-0.35 * * \\
(0.14)\end{array}$ \\
\hline 2010 & $\begin{array}{r}0.07 * * * \\
(0.02)\end{array}$ & & & $\begin{array}{l}0.05 \\
(0.04)\end{array}$ & & & $\begin{array}{r}0.64 * * * \\
(0.08)\end{array}$ & & \\
\hline $\mathrm{GC}$ & $\begin{array}{l}0.04 \\
(0.03)\end{array}$ & $\begin{array}{l}0.02 \\
(0.05)\end{array}$ & $\begin{array}{r}0.08^{* *} \\
(0.03)\end{array}$ & $\begin{array}{r}-0.61 * * * \\
(0.06)\end{array}$ & $\begin{array}{r}-0.60 * * * \\
(0.08)\end{array}$ & $\begin{array}{r}-0.64 * * * \\
(0.07)\end{array}$ & $\begin{array}{r}0.95 * * * \\
(0.17)\end{array}$ & $\begin{array}{r}0.91^{* * * *} \\
(0.30)\end{array}$ & $\begin{array}{r}0.95 * * * \\
(0.22)\end{array}$ \\
\hline Constante & $\begin{array}{r}4.34 * * * \\
(0.02)\end{array}$ & $\begin{array}{r}4.32 * * * \\
(0.03)\end{array}$ & $\begin{array}{r}4.43^{* * *} \\
(0.03)\end{array}$ & $\begin{array}{r}3.29 * * * \\
(0.04)\end{array}$ & $\begin{array}{r}3.33 * * * \\
(0.05)\end{array}$ & $\begin{array}{r}3.31 * * * \\
(0.04)\end{array}$ & $\begin{array}{r}1.83 * * * * \\
(0.09)\end{array}$ & $\begin{array}{r}1.71 \text { *** } \\
(0.13)\end{array}$ & $\begin{array}{r}2.54 * * * \\
(0.08)\end{array}$ \\
\hline
\end{tabular}

Nota: esta tabla muestra los resultados de la estimación de las ecuaciones, con errores estándares robustos para aislar el efecto de la presencia de heterocedasticidad detectada. Cada modelo cuenta con el coeficiente asociado a la variable, con asteriscos mostrando el nivel de significancia estadística asociado; “*” denota significancia al 10\%, “**” al 5\% y “***” al $1 \%$. Debajo de las variables asociadas a la luminosidad se encuentra el término "->GC", que indica la interacción de la variable superior con la variable dicotómica que toma valor 1 cuando el municipio pertenece a Santo Domingo, Santiago o el Distrito Nacional.

\section{Pobreza}

La tabla debajo muestra la estimación de las diferentes ecuaciones pertinentes a la pobreza, mostrando el efecto de la separación de la muestra por años. El modelo conjunto del grupo socioeconómico muy bajo más bajo se deteriora cuando se compara la estimación del año 2010 contra la estimación sobre la muestra de 2002; el coeficiente asociado al cuadrado del promedio de luminosidad pierda totalmente su significancia estadística, mientras que el efecto de la desviación estándar se reduce (coeficiente pasa de -0.08 a -0.07 y significancia estadística disminuye). En el caso de los modelos separados de los grupos muy bajo y bajo, la significancia se mantiene relativamente 
igual para la mayoría de las variables, con los coeficientes sin cambios significativos.

Tabla 10. Prueba de robustez por años de modelos de pobreza

\begin{tabular}{|c|c|c|c|c|c|c|c|c|c|}
\hline & \multicolumn{3}{|c|}{ GSE - Muy Bajo } & \multicolumn{3}{|c|}{ GSE - Bajo } & \multicolumn{3}{|c|}{ GSE - Muy Bajo + Bajo } \\
\hline & $\begin{array}{l}\text { Total } \\
\text { (1) }\end{array}$ & $\begin{array}{c}2002 \\
(2) \\
\end{array}$ & $\begin{array}{c}2010 \\
(3)\end{array}$ & $\begin{array}{c}\text { Total } \\
(4)\end{array}$ & $\begin{array}{c}2002 \\
(5) \\
\end{array}$ & $\begin{array}{c}2010 \\
(6) \\
\end{array}$ & $\begin{array}{c}\text { Total } \\
(7)\end{array}$ & $\begin{array}{c}2002 \\
(8) \\
\end{array}$ & $\begin{array}{c}2010 \\
(9)\end{array}$ \\
\hline Ln(Media) & $\begin{array}{r}-0.20^{* * * *} \\
(0.07)\end{array}$ & $\begin{array}{c}-0.17^{*} \\
(0.09)\end{array}$ & $\begin{array}{c}-0.23^{*} \\
(0.12)\end{array}$ & $\begin{array}{r}0.08^{* * * *} \\
(0.02)\end{array}$ & $\begin{array}{r}0.07^{* * *} \\
(0.03)\end{array}$ & $\begin{array}{r}0.08^{* *} \\
(0.04)\end{array}$ & $\begin{array}{c}-0.02 \\
(0.03)\end{array}$ & $\begin{array}{c}-0.01 \\
(0.04)\end{array}$ & $\begin{array}{c}-0.02 \\
(0.06)\end{array}$ \\
\hline$->\mathrm{GC}$ & $\begin{array}{c}0.48^{* *} \\
(0.20)\end{array}$ & $\begin{array}{l}0.58 \\
(0.37)\end{array}$ & $\begin{array}{l}0.45^{* *} \\
(0.23)\end{array}$ & $\begin{array}{l}0.05 \\
(0.11)\end{array}$ & $\begin{array}{l}0.14 \\
(0.14)\end{array}$ & $\begin{array}{l}0.06 \\
(0.15)\end{array}$ & $\begin{array}{l}0.18 \\
(0.12)\end{array}$ & $\begin{array}{l}0.27 \\
(0.20)\end{array}$ & $\begin{array}{l}0.16 \\
(0.16)\end{array}$ \\
\hline Ln(Media)'Sqr & $\begin{array}{c}-0.03 \\
(0.02)\end{array}$ & $\begin{array}{l}-0.03 \\
(0.02)\end{array}$ & $\begin{array}{l}-0.03 \\
(0.03)\end{array}$ & $\begin{array}{r}-0.03 * * * \\
(0.01)\end{array}$ & $\begin{array}{r}-0.04 * * * \\
(0.01)\end{array}$ & $\begin{array}{r}-0.03 * * \\
(0.01)\end{array}$ & $\begin{array}{r}-0.02 * * * \\
(0.01)\end{array}$ & $\begin{array}{r}-0.03^{* *} \\
(0.01)\end{array}$ & $\begin{array}{c}-0.02 \\
(0.02)\end{array}$ \\
\hline$->\mathrm{GC}$ & $\begin{array}{r}-0.12^{* * * *} \\
(0.04)\end{array}$ & $\begin{array}{c}-0.14^{*} \\
(0.07)\end{array}$ & $\begin{array}{r}-0.12 * * \\
(0.05)\end{array}$ & $\begin{array}{c}-0.04 * \\
(0.02)\end{array}$ & $\begin{array}{c}-0.06^{*} \\
(0.03)\end{array}$ & $\begin{array}{c}-0.05 \\
(0.04)\end{array}$ & $\begin{array}{r}-0.07^{* * * *} \\
(0.03)\end{array}$ & $\begin{array}{r}-0.08^{* *} \\
(0.04)\end{array}$ & $\begin{array}{r}-0.07^{*} \\
(0.04)\end{array}$ \\
\hline Ln(Std. Dev.) & $\begin{array}{r}-0.13^{* *} \\
(0.06)\end{array}$ & $\begin{array}{r}-0.18^{* *} \\
(0.08)\end{array}$ & $\begin{array}{l}-0.08 \\
(0.09)\end{array}$ & $\begin{array}{r}-0.08^{* * * *} \\
(0.02)\end{array}$ & $\begin{array}{c}-0.05^{*} \\
(0.03)\end{array}$ & $\begin{array}{r}-0.10^{* * * *} \\
(0.03)\end{array}$ & $\begin{array}{r}-0.12 * * * \\
(0.03)\end{array}$ & $\begin{array}{r}-0.13 * * * \\
(0.03)\end{array}$ & $\begin{array}{r}-0.10^{* *} \\
(0.04)\end{array}$ \\
\hline$->\mathrm{GC}$ & $\begin{array}{l}0.01 \\
(0.14)\end{array}$ & $\begin{array}{l}-0.05 \\
(0.29)\end{array}$ & $\begin{array}{l}0.01 \\
(0.12)\end{array}$ & $\begin{array}{c}0.12^{*} \\
(0.07)\end{array}$ & $\begin{array}{l}0.04 \\
(0.13)\end{array}$ & $\begin{array}{l}0.16^{*} \\
(0.09)\end{array}$ & $\begin{array}{l}0.12 \\
(0.09)\end{array}$ & $\begin{array}{l}0.05 \\
(0.17)\end{array}$ & $\begin{array}{l}0.13 \\
(0.09)\end{array}$ \\
\hline 2010 & $\begin{array}{r}-0.34 * * * \\
(0.05)\end{array}$ & & & $\begin{array}{r}-0.23 * * * \\
(0.02)\end{array}$ & & & $\begin{array}{r}-0.27 * * * \\
(0.02)\end{array}$ & & \\
\hline $\mathrm{GC}$ & $\begin{array}{r}-0.46^{* * * *} \\
(0.10)\end{array}$ & $\begin{array}{l}-0.36 \\
(0.23)\end{array}$ & $\begin{array}{r}-0.51^{* * * *} \\
(0.10)\end{array}$ & $\begin{array}{r}-0.23 * * * \\
(0.08)\end{array}$ & $\begin{array}{l}-0.07 \\
(0.11)\end{array}$ & $\begin{array}{r}-0.38 * * * \\
(0.07)\end{array}$ & $\begin{array}{r}-0.35^{* * * *} \\
(0.07)\end{array}$ & $\begin{array}{c}-0.22^{*} \\
(0.13)\end{array}$ & $\begin{array}{r}-0.45^{\text {**** }} \\
(0.06)\end{array}$ \\
\hline Constante & $\begin{array}{r}3.26 * * * \\
(0.05) \\
\end{array}$ & $\begin{array}{r}3.27^{* * *} \\
(0.06) \\
\end{array}$ & $\begin{array}{r}2.92^{* * * *} \\
(0.06) \\
\end{array}$ & $\begin{array}{r}3.72^{* * *} \\
(0.02) \\
\end{array}$ & $\begin{array}{r}3.70^{* * *} \\
(0.03) \\
\end{array}$ & $\begin{array}{r}3.51^{* * *} \\
(0.02) \\
\end{array}$ & $\begin{array}{r}4.25^{* * *} \\
(0.02) \\
\end{array}$ & $\begin{array}{r}4.24 * * * \\
(0.02) \\
\end{array}$ & $\begin{array}{r}3.98^{* * * *} \\
(0.03) \\
\end{array}$ \\
\hline
\end{tabular}

Nota: esta tabla muestra los resultados de la estimación de las ecuaciones, con errores estándares robustos para aislar el efecto de la presencia de heterocedasticidad detectada. Cada modelo cuenta con el coeficiente asociado a la variable, con asteriscos mostrando el nivel de significancia estadística asociado; "*” denota significancia al 10\%, “**” al 5\% y “***” al $1 \%$. Debajo de las variables asociadas a la luminosidad se encuentre el término "->GC", que indica la interacción de la variable superior con la variable dicotómica que toma valor 1 cuando el municipio pertenece a Santo Domingo, Santiago o el Distrito Nacional.

\section{Índice de Calidad de Vida}

En la siguiente tabla se muestra que la estimación del modelo de Índice de Calidad de Vida no experimenta cambios importantes cuando se realiza en cada año de la muestra por separado. Sin embargo, es importante notar que el coeficiente más significativo dentro del modelo estimado, que es el asociado a la desviación estándar de la luminosidad dentro de los municipios, tiene un valor de 0.05 en 2010, habiendo disminuido desde 0.08 en la estimación de 2002. Esto apoya el hallazgo en los modelos anteriormente verificados de que es posible que en la medida que transcurran los años el poder predictivo de la luminosidad sobre algunas variables disminuya. 

Tabla 11. Prueba de robustez por años de modelos
de Í Ínice de Calidad de Vida

\begin{tabular}{|c|c|c|c|}
\hline & \multicolumn{3}{|c|}{ ICV } \\
\hline & $\begin{array}{l}\text { Total } \\
\text { (1) }\end{array}$ & $\begin{array}{c}2002 \\
(2)\end{array}$ & $\begin{array}{c}2010 \\
(3)\end{array}$ \\
\hline Ln(Media) & $\begin{array}{c}0.004 \\
(0.02)\end{array}$ & $\begin{array}{l}0.01 \\
(0.02)\end{array}$ & $\begin{array}{c}-0.01 \\
(0.03)\end{array}$ \\
\hline$->\mathrm{GC}$ & $\begin{array}{r}-0.05^{*} \\
(0.03)\end{array}$ & $\begin{array}{l}-0.07 \\
(0.04)\end{array}$ & $\begin{array}{c}-0.04 \\
(0.03)\end{array}$ \\
\hline Ln(Media)'Sqr & $\begin{array}{l}0.01 \\
(0.00)\end{array}$ & $\begin{array}{c}0.004 \\
(0.01)\end{array}$ & $\begin{array}{c}0.01 * \\
(0.01)\end{array}$ \\
\hline$->\mathrm{GC}$ & $\begin{array}{r}0.02 * * * \\
(0.01)\end{array}$ & $\begin{array}{r}0.02 * * \\
(0.01)\end{array}$ & $\begin{array}{c}0.02 * * \\
(0.01)\end{array}$ \\
\hline Ln(Std. Dev.) & $\begin{array}{r}0.07 * * * \\
(0.01)\end{array}$ & $\begin{array}{r}0.08 * * * \\
(0.02)\end{array}$ & $\begin{array}{r}0.05^{* * * *} \\
(0.02)\end{array}$ \\
\hline$->\mathrm{GC}$ & $\begin{array}{c}-0.04 \\
(0.02)\end{array}$ & $\begin{array}{l}-0.04 \\
(0.04)\end{array}$ & $\begin{array}{c}-0.03 \\
(0.03)\end{array}$ \\
\hline 2010 & $\begin{array}{r}0.21 * * * \\
(0.01)\end{array}$ & & \\
\hline $\mathrm{GC}$ & $\begin{array}{r}0.07 * * * \\
(0.02)\end{array}$ & $\begin{array}{r}0.08 * * \\
(0.03)\end{array}$ & $\begin{array}{r}0.07 * * * \\
(0.02)\end{array}$ \\
\hline Constante & $\begin{array}{r}3.83 * * * \\
(0.01)\end{array}$ & $\begin{array}{r}3.82^{* * * *} \\
(0.02)\end{array}$ & $\begin{array}{r}4.05^{* * *} \\
(0.01)\end{array}$ \\
\hline
\end{tabular}

Nota: esta tabla muestra los resultados de la estimación de las ecuaciones, con errores estándares robustos para aislar el efecto de la presencia de heterocedasticidad detectada. Cada modelo cuenta con el coeficiente asociado a la variable, con asteriscos mostrando el nivel de significancia estadística asociado; "*” denota significancia al 10\%, “**” al 5\% y "***" al 1\%. Debajo de las variables asociadas a la luminosidad se encuentre el término "->GC", que indica la interacción de la variable superior con la variable dicotómica que toma valor 1 cuando el municipio pertenece a Santo Domingo, Santiago o el Distrito Nacional.

\section{Conclusión}

En esta investigación se comprobó la relación entre la presencia de luces nocturnas y diversas variables demográficas. Se encontraron relaciones significativas entre la luminosidad y la población, la densidad poblacional, el acceso al tendido eléctrico, la pobreza, el hacinamiento, el Índice de Calidad de Vida y el saneamiento.

Estos hallazgos van en consonancia con la literatura internacional y abren espacio en la República Dominicana para estudios posteriores 
que sirvan de complemento a la producción de estadísticas demográficas actualizadas, la actualización de los marcos muestrales de los censos (para parametrizar mejor las encuestas oficiales en años donde no ocurren censos) y la identificación de cambios en las tendencias de indicadores de interés, como la pobreza en tiempo real.

Para poder lograr las iniciativas mencionadas anteriormente se hace necesario desarrollar tres líneas de estudio complementarias. Primero, debido a que las fotos satelitales de la NOAA cambian de metodología de recolección a partir de 2012, pero ganan la ventaja de una periodicidad mensual en vez de anual, es necesario desarrollar una metodología de empalme de las series de promedio y desviación estándar de la luminosidad por municipio. Segundo, es necesario explorar metodologías de inferencia estadística más robustas, como la utilización de redes neurales, para aproximar de manera más precisa las relaciones entre la luminosidad y los diferentes indicadores sociodemográficos. Tercero, la utilización de estas informaciones para actualizar las estadísticas demográficas (con énfasis en las de pobreza) y los marcos muestrales del censo requiere de un nivel de ajuste estadístico superior al que resultó en la serie de ecuaciones estimadas.

Para lograr lo anterior es posible que sea necesario complementar las variables explicativas relacionadas a la luminosidad con fuentes de datos alternativas que cumplan con las condiciones de periodicidad mensual, bajo rezago de actualización, granularidad, al menos provincial, y alto poder predictivo sobre las variables demográficas.

\section{Referencias bibliográficas}

Addison, D. \& Stewart, B. (2015). Nighttime Lights Revisited: The Use of Nighttime Lights Data as a Proxy for Economic Variables. World Bank Policy Research Working Paper 7496. Recuperado de http://hdl.handle.net/10986/23460

Amaral, S., Camara, G., Monteiro, A., Quintanilha, J. \& Elvidge, C. (2005). Estimating population and energy consumption in Brazilian Amazonia using DMSP night-time satellite data. 
Computers, Environment and Urban Systems, 29, 179-195. DOI:10.1016/j.compenvurbsys.2003.09.004

Breusch, T. \& Pagan, A. (1980). The Lagrange Multiplier Test and its Applications to Model Specification in Econometrics. Review of Economic Studies, 47(1), 239-253. DOI:10.2307/2297111

Doll, C., Muller, J. \& Elvidge, C. (2000). Night-time Imagery as a Tool for Global Mapping of Socioeconomic Parameters and Greenhouse Gas Emissions. AMBIO A Journal of the Human Environment, 29(3), 157-162. DOI: 10.1579/0044-744729.3.157

Donaldson, D. \& Storeygard, A. (2016). The View from Above, Applications of Satellite Data in Economics. Journal of Economic Perspectives, 30(4), 171-198. DOI:10.1257/jep.30.4.171

Elvidge, C., Baugh, K., Kihn, E., Kroehl, H., Davis, E. \& Davis, C. (1997). Relation between satellite observed visible-near infrared emissions, population, economic activity and electric power consumption. International Journal of Remote Sensing, 18(6), 1373-1379. DOI:10.1080/014311697218485

Elvidge, C., Imhoff, M., Baugh, K., Hobson, V., Nelson, I., Safran, J., Dietz, J., \& Tuttle, B. (2001). Night-time lights of the world: 1994-1995. ISPRS Journal of Photogrammetry and Remote Sensing, 56(2), 81-99. DOI:10.1016/S0924-2716(01)00040-5

Elvidge, C., Baugh, K., Zhizhin, M. \& Chi, F. (2013). Why VIRRS data are superior to DMSP for mapping nighttime lights. Proceedings of the Asia-Pacific Advanced Network, 35, 62-69. DOI:10.7125/APAN.35.7

Ghosh, T., Anderson, S., Powell, R., Sutton, P. \& Elvidge, C. (2009). Estimation of Mexico's Informal Economy and Remittances Using Nighttime Imagery. Remote Sensing, 1, 418-444. DOI:10.3390/rs1030418

Henderson, J., Storeygard, A. \& Weil, D. (2012). Measuring Economic Growth from Outer Space. American Economic Review, 102(2), 994-1028. DOI:10.1257/aer.102.2.994

Roychowdhury, K., Jones, S., Arrowsmith, C., Reinke, K. \& Bedford, A. (2010). The role of satellite data in census: Case study of 
an Indian State. Proceedings of the Asia-Pacific Advanced Network, 30, 207-218. DOI:10.7125/APAN.30.23

Sutton, P., Roberts, D., Elvidge, C. \& Meij, H. (1997). A Comparison of Nighttime Satellite Imagery and Population Density for the Continental United States. Photogrammetric Engineering and Remote Sensing, 63(11), 1303-1313. DOI:0099-1112/97/6311$1303 \$ 3.00 / 0$ 